\title{
Pyloric stenosis in Western Australia, 1971-84
}

\author{
N E HITCHCOCK, A I GILMOUR, M GRACEY, AND V BURKE \\ Princess Margaret Children's Medical Research Foundation and Department of Child Health, University of \\ Western Australia, Perth, Western Australia
}

\begin{abstract}
SUMmaRY Analyses of hospital records and census data for 1971-84 showed no significant increase in incidence of infantile hypertrophic pyloric stenosis in Western Australia. No link was found between breast feeding and incidence of disease. Low birth weight, short gestation pregnancies, and paternal family history of the disease were significant features.
\end{abstract}

The incidence of infantile hypertrophic pyloric stenosis is reportedly increasing in some areas. ${ }^{1}$ It has been suggested that the reported increase is related to changes in breast feeding practices. $^{2}$

The Western Australian population of 1.4 million (over $70 \%$ of whom live in Perth) is uniquely isolated geographically. The State Health Department has full coverage of all hospital morbidity and surgical procedures in the state. This allows analysis of the state wide incidence of pyloric stenosis and possible associated factors, including infant feeding practices, birth weight, birth order, and sex ratio.

\section{Patients and methods}

Information about all patients in Western Australia who between 1971 and 1984 were diagnosed as having pyloric stenosis and were treated surgically was provided from the hospital morbidity collection of the State Health Department. Records of all patients who underwent operation for pyloric stenosis in Princess Margaret Hospital in the same period were examined personally by the authors.

Data were analysed using log linear models and linear regression with SPSS/PC.

\section{Results and discussion}

During the 14 year period 1971-84, 495 boys and 107 girls with pyloric stenosis were treated surgically in Princess Margaret Hospital, Perth. These 602 patients formed $97 \%$ of those with this disease operated on in Western Australia over that period.

Most features of the disease found in our cohort were typical of the established features of pyloric stenosis. These included pronounced male pre- ponderance, clinical features, and mean age at diagnosis. The mean age at diagnosis was 5.5 weeks. The male:female ratio (4.9) was significant $(p<0.05)$. The incidence per 1000 live births varied from a low of 1.4 in 1972 to a high of 2.9 in 1977 (Figure), with the male rate ranging from $1 \cdot 6 / 1000$ in 1972 to $4 \cdot 5 / 1000$ in 1978 and the female rate from $0 \cdot 4 / 1000$ in 1976 to $1 / 1000$ in 1975 . The rate varied considerably in single year periods and in 4-5 year periods from $1 \cdot 7 / 1000(1971-74)$ to $2 \cdot 4 / 1000$ (197579). This variation has been noted by previous workers. ${ }^{1}$

The slope of the regression line for the data on pyloric stenosis was not significantly different from zero, indicating that there was no significant increase in the incidence of the disease over the 14 years.

Although the rate of breast feeding in Western Australia on leaving hospital increased from about $45 \%$ in 1971 to more than $80 \%$ in 1984 , there was no significant difference in rates of breast feeding in infants with pyloric stenosis and in controls (Figure). Regression analysis showed the rate of change in breast feeding during the study to be significantly different from the rate of change in the disease $(p<0 \cdot 05)$. This finding argues against changes in infant feeding patterns influencing the frequency of this disease.

Five other features in our cohort are worthy of note:

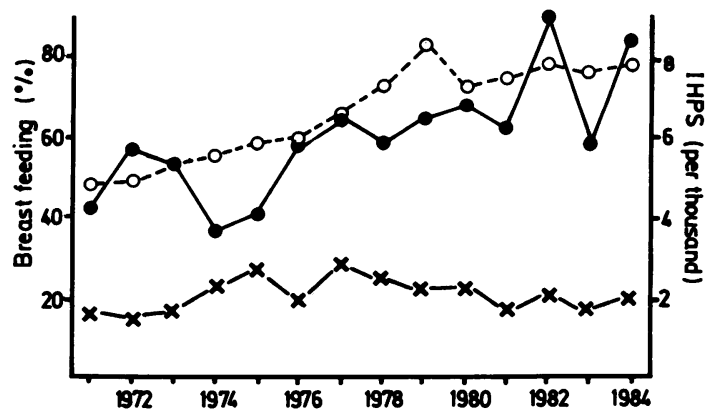

Figure Incidence of infantile hypertrophic pyloric stenosis (IHPS) $(\times-\times)$ and of breast feeding in mothers of patients with pyloric stenosis (-) and mothers of controls (O- - ), 1971-84. 
(1) Birth order had no significant influence on the incidence of the disease in boys or girls. Birth order distribution (first born, $42.9 \%$; second, $31.8 \%$, third, $17.9 \%$; fourth, or more $7 \cdot 4 \%$ ) did not differ significantly from census data. ${ }^{3}$

(2) There were significantly more infants with low birth weight $(<2500 \mathrm{~g})(9.3 \%)$ in the group with pyloric stenosis than in the control population $(4.9 \%){ }^{4}$

(3) Short gestation pregnancies ( $<38$ weeks) occurred in $16 \%$ of mothers of patients with pyloric stenosis (excluding multiple births); this compares with $9 \%$ in the general Western Australian population. (Hitchcock NE. Unpublished data.)

(4) Several Aboriginal patients had the disease. To our knowledge, pyloric stenosis has not previously been reported in Australian Aborigines. Unfortunately, the hospital records did not describe how many of these Aboriginal patients were of full descent or were part Aboriginal.

(5) A family history of pyloric stenosis was present in 65 patients $(11 \%)$, just over half being in the father's family, one quarter in the mother's family, and the rest in siblings. There were 13 multiple births involved. In only two instances were both infants affected, and in one set of all male triplets only one infant was affected. Pyloric stenosis has been shown, in other studies, to be significantly more common in children of affected parents, especially if the affected parent is the mother ${ }^{5} 6$ -perhaps because the gene responsible for the disease in girls has greater penetrance than that responsible for the disease in boys. This was not our experience in Perth.

We are grateful to the medical records staff of Princess Margaret Hospital for their cooperation and to the Health Department of Western Australia and the Australian Bureau of Statistics for statistical information.

\section{References \\ 1 Anonymous. Incidence of infantile hypertrophic pyloric steno- sis [Editorial]. Lancet. 1984; i:888-9. \\ 2 Webb AR, Lari J, Dodge JA. Infantile hypertrophic pyloric stenosis in South Glamorgan 1970-9. Effects of changes in feeding practice. Arch Dis Child 1983;58:586-90. \\ 3 Australian Bureau of Statistics. Australia Year Book No 66. Canberra: Australian Bureau of Statistics, 1982. \\ 4 Western Australian Midwives Notification System. Perinatal statistics in Western Australia. Second annual report of the Western Australian Midwives Notification System for 1984 Perth: Health Department of Western Australia, 1986. \\ 5 McKeown T, MacMahon B. Infantile hypertrophic pyloric stenosis in parent and child. Arch Dis Child 1955;30:497-500. \\ 6 Carter CO. The inheritance of congenital pyloric stenosis. $\mathrm{Br}$ Med Bull 1961;17:251-4.}

Correspondence to Mrs N E Hitchcock, GPO Box D184, Perth, 6001, Australia.

Received 14 November 1986
SUMMARY A child with idiopathic pulmonary haemosiderosis for three years required three monthly transfusions. Circulating avian, gliadin, and reticulin antibodies suggested the diagnosis of gluten enteropathy, and jejunal biopsy showed subtotal villous atrophy. During 15 months on a gluten free diet his growth and behaviour improved and he required no transfusions.

Pulmonary haemosiderosis is characterised by recurrent pulmonary infiltrates due to alveolar haemorrhage, anaemia, and finding haemosiderin laden macrophages in the sputum. In adults certain systemic disorders may be associated, but in chil- dren no specific cause is usually found. A proportion, however, have circulating antibodies to cow's milk proteins, and withdrawal of cow's milk from the diet may be beneficial. ${ }^{1}$

\section{Case report}

The boy was born at term weighing $2490 \mathrm{~g}$ and was formula fed. At 2 years of age his height and weight lay just below the 3rd centile.

He presented aged 4.5 years with a few days' history of pallor and decline in his chronically poor appetite. His height $(1.06 \mathrm{~m})$ and weight $(15.5 \mathrm{~kg})$ still lay just below the 3rd centile. An initial haemoglobin concentration of $5.2 \mathrm{~g} / \mathrm{dl}$ was attributed to iron deficiency, but an erythrocyte sedi- 